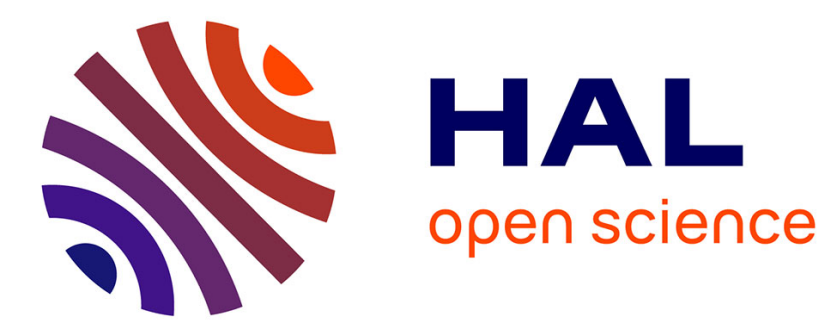

\title{
Déraison de Paris et raison d'État dans la Satyre Menippee (1593-1595)
}

Audrey Duru

\section{To cite this version:}

Audrey Duru. Déraison de Paris et raison d'État dans la Satyre Menippee (1593-1595). Stéphane BOURDIN; Anne RELTGEN-TALLON; Michel PAOLI. La Forme de la ville de l'Antiquité à la Renaissance, Presses universitaires de Rennes, pp.221-239, 2015, 978-2-7535-3625-8. hal-01216830

\section{HAL Id: hal-01216830 \\ https://hal.science/hal-01216830}

Submitted on 17 Oct 2015

HAL is a multi-disciplinary open access archive for the deposit and dissemination of scientific research documents, whether they are published or not. The documents may come from teaching and research institutions in France or abroad, or from public or private research centers.
L'archive ouverte pluridisciplinaire HAL, est destinée au dépôt et à la diffusion de documents scientifiques de niveau recherche, publiés ou non, émanant des établissements d'enseignement et de recherche français ou étrangers, des laboratoires publics ou privés. 
«Déraison de Paris et raison d'État dans la Satyre Menippee (1593-1595) », dans : La Forme de la ville de l'Antiquité à la Renaissance, actes du colloque international d'Amiens, 7-9 novembre 2011, dir. Stéphane Bourdin, Anne Reltgen-Tallon et Michel PaOli, Rennes, Presses universitaires de Rennes, 2015, p. 221-239.

\section{Déraison de Paris et raison d'État dans la Satyre Menippee (1593-1595)}

\section{Audrey Duru}

Dénoncer l'imposture d'un discours de sédition, énoncer une parole de vérité adéquate à la justice, voilà les fonctions dévolues au libelle qu'est la Satyre Menippee ${ }^{1}$. À travers la mise en scène du conflit des éloquences et opinions à Paris en 1593, la Satyre Menippee vise en effet à concurrencer la Ligue sur son propre terrain, l'enrôlement des délégués aux états généraux, et à fléchir les hésitants en faveur de la paix. Dans ce conflit oratoire transposé sur papier, Paris est un «mot valeur ${ }^{2} »$ et la forme de la ville, un motif d'orgueil.

Le recueil fait partie des textes polémiques et satiriques que le moment de la Ligue a produits, en particulier à Paris, dans le contexte d'une intense campagne de presse(s) ${ }^{3}$. Diffusé en manuscrit à Paris dès avril ou mai 1593, le libelle est imprimé à Tours l'année suivante, puis dans d'autres villes, parfois avec la fausse date de 1593. Selon une lecture prédominante, du moins depuis les études que la Satyre Menippee a suscitées au XIX ${ }^{\mathrm{e}}$ siècle, le libelle défend, contre le «catholicon » ou drogue espagnole, un intérêt français. Cependant, la défense de l'intérêt national contient un tiroir, une défense de l'intérêt de Paris.

La dernière harangue du libelle ${ }^{4}$ offre apparemment la parole sérieuse qui commande la dénonciation d'ensemble par la parodie et le burlesque. Les valeurs qui la sous-tendent se rapportent à la forme constitutive de l'identité de Paris : place forte ; cité où par les orateurs et imprimeurs se constitue un public (à défaut encore d'une opinion publique ${ }^{5}$ ); ville franche pourvue de privilèges, au moins dans le passé. Cependant, la défense nostalgique des valeurs municipales et parisiennes sert paradoxalement la désignation d'un pouvoir souverain et absolu : le roi naturel. La dernière harangue, attribuée fictivement à un certain Claude

\footnotetext{
${ }^{1}$ Le titre de cette collection de textes est instable, tout comme sa composition au fil des impressions. Par commodité et suivant l'usage établi, nous utilisons le titre abrégé de Satyre Menippee. Dans cette étude, la graphie Satyre Menippee, sans accents conformément à l'usage du XVI siècle, désigne donc le libelle, tandis que «satyre ménippée », avec accents graphiques, désigne le genre littéraire connu sous ce nom. Édition de référence : Satyre Menippee de la vertu du catholicon d'Espagne et de la tenue des Estats de Paris, établie par M. MARTIN d'après une impression sous ce titre de 1595, Paris, Champion, 2007, bibliographie p. 661-750. Complément bibliographique depuis 2007 : SZABARI A., «Menippean Satire and the Typographic Space of Paris », Less Rightly Said. Scandals and Readers in Sixteenth-Century France, Stanford, Stanford University Press, 2010, p. 175-184.

2 ANGENOT M., La Parole pamphlétaire. Contribution à la typologie des discours modernes, Paris, Payot, 1982, p. 131-136 : il nomme «mot valeur » un «mot marqué axiologiquement ». La première attestation en français de l'anglicisme pamphlet daterait de 1653 : voir BELLENGER Y., «Le pamphlet avant le pamphlet: le mot et la chose », CAIEF, 1984, n 36, p. 87-96, et Le Pamphlet en France au XVI ${ }^{\mathrm{e}}$ siècle, Paris, École normale supérieure de jeunes filles, 1983. En revanche, «libelle accusatoire» est attesté dans le dictionnaire latin-français d'Estienne (1552) sous l'entrée instituere accusationem, et «libelle diffamatoire » dans le Thresor de Nicot (1606). Voir aussi Jouhaud C., «Les libelles en France dans le premier XVII siècle: lecteurs, auteurs, commanditaires et historiens », XVII siècle, «La littérature pamphlétaire à l'âge classique », $\mathrm{n}^{\mathrm{o}}$ 195, avril-juin 1997, p. 203-217.

${ }^{3}$ PALLIER D., Recherches sur l'imprimerie à Paris pendant la Ligue, 1585-1594, Genève, Droz, 1975 ; sur la figuration de Paris dans les libelles, voir p. 183-186. Sur l'histoire de la Ligue à Paris, voir les travaux d'Élie Barnavi, Frederic J. Baumgartner, Jean-Marie Constant, Denis Crouzet, Robert Descimon.

${ }_{5}^{4}$ Éd. cit., p. 75-134.

${ }^{5}$ JOUHAUD C., art. cit., p. 208-209.
} 
d'Aubray, relève elle-même de l'esthétique du mélange propre au genre de la «satyre ménippée ${ }^{6} \gg$. Cette harangue assemble différents discours que nous étudierons successivement: éloquence municipale préoccupée du bien commun urbain, discours de doléances aux états généraux, imagerie ancienne de la royauté. Il en résulte un discours théologico-politique composite, caractéristique des partisans sans parti souvent qualifiés de « Politiques ».

Nous montrerons que ce qui charpente la harangue de d'Aubray et le libelle dans son ensemble, ce n'est pas l'adhésion à un parti mais l'idée d'État, qu'incarne effectivement, selon les rédacteurs du libelle, la personne du roi naturel, Henri de Navarre. L'éloquence démystificatrice est celle de la doctrine de l'État fondé en droit naturel, bref, une éloquence de la « raison d'État».

\section{Le public parisien et la voix de l'hôtel de ville}

La Satyre Menippee est tenue pour l'expression des Politiques ${ }^{7}$ dans la mesure où elle défend une solution de paix. L'historiographie a cependant mis en évidence les difficultés que soulève la notion de Politiques ${ }^{8}$. Or, face à la crise ouverte à partir de 1585 par la contestation des Princes ligueurs menés par les Guises et de quelques villes, si la Satyre Menippee offre une issue, c'est précisément parce qu'elle n'adopte pas la voix d'un «parti partisan » ${ }^{9}$. Le compromis défendu et exposé à travers un discours de fiction aux états généraux est prêté à un délégué parisien. Ce dernier propose une alliance locale entre la bonne ville de Paris et le roi, tout en appelant les autres villes à suivre cet exemple.

\section{La purgation des passions dans l'éloquence délibérative}

Selon le volume d'Études sur la Satyre Menippee paru en $1987^{10}$, le moment satirique de la Satyre Menippee ou Catholicon (« remède universel »), grâce au travestissement comique des discours ligueurs, purge l'éloquence politique et la prédication des clercs qui ont accompagné l'insurrection intestine de Paris.

Conformément au genre de la «satyre ménippée », cette collection de textes forme une œuvre bigarrée. Elle mêle tonalité comique et tonalité sérieuse et, en outre, alterne prose et vers. La harangue de d'Aubray est elle-même un prosimètre polyphonique, qui fait entendre, par des citations d'épigrammes ou de chansons qui courent les rues, la voix du peuple parisien. Toutefois, le peuple se contente de répéter un texte le plus souvent attribué à un rédacteur

\footnotetext{
${ }^{6}$ Dans la littérature latine, la satura désigne un mélange culinaire, puis une œuvre caractérisée par la diversité des formes, genres, thèmes, tons, etc. «Satire (ou satyre) ménippée » est un emprunt à un titre de Varron par Juste Lipse : à partir de 1581, la satyre ménippée devient un genre de texte. Voir dans l'édition de référence le second «discours de l'Imprimeur », introduit en 1595, p. 160-161, ainsi que DE SMET I., Menippean Satire and the Republic of Letters 1581-1655, Genève, Droz, 1996 ; NEDELEC C., « Le politique travesti : l'influence de la Satyre Ménippée au XVII siècle », J. BERChTOLD et M.-M. FrAGONARD (dir.), La Mémoire des guerres de religion. La concurrence des genres historiques (XVI $I^{e}$ XVIII siècle), Genève, Droz, 2007, p. 175-192.

7 Sur ce mot, voir DEMONET M.-L., «Quelques avatars du mot "politique" (XIV -XVII" siècle) », Langage et société, $\mathrm{n}^{\mathrm{o}} 113,2005-3$, p. 33-61.

${ }^{8}$ Bettinson C., « The Politiques and the Politique Party: A Reappraisal », K. CAMERon (dir.), From Valois to Bourbon: Dynasty, State and Society in Early Modern France, Exeter, Exeter University Press, 1989, p. 35-49; TURChetTi M., «Une question mal posée : l'origine et l'identité des Politiques au temps des guerres de Religion », Th. WANEGFFELEN (dir.), De Michel de L'Hospital à l'édit de Nantes. Politique et religion face aux Églises, Clermont-Ferrand, Presses universitaires Blaise-Pascal, 2002, p. 357-390.

9 FRAGONARD M.-M., «Donner toute priorité à la paix du royaume : un argument des Politiques ?», ibid., p. 419-438, ici p. 420.

${ }^{10}$ Lestringant F., Menager D. (dir.), Études sur la Satyre Ménippée, Genève, Droz, 1987. Nous avons particulièrement utilisé les articles suivants : MENAGER D., «La crise de l'éloquence », p. 121-149, et «Dieu et le roi », p. 201-226 ; VIGNES J., « Culture et histoire dans la Satyre Menippee », p. 151-199 ; BARRACHINA M.A., GOMEZ-GERAUD M.-C., «L'esprit national : pour une monarchie restaurée ou instaurée ? », p. $227-274$.
} 
prétendu : par exemple, d'Aubray cite un « quatrain maintenant tout vulgaire » qu'il attribue à François I $^{\mathrm{er}}$ (p. 84). De même, lorsque d'Aubray cite des vers qu'il a « retenu[s] [...] par cœur, par ce qu'ils sont si vulgaires, que les femmes et petits enfans les ont appris, et qu'il ne se peut rien faire de plus naif pour exprimer nos procedures, et les façons dont nous avons usé pour trouvé de l'argent » (p. 98), les vers deviennent ambigus : sont-ils une dénonciation par l'antiphrase ironique de la rhétorique ligueuse ou au contraire la diffusion, par la voix des simples, d'un appel au pillage sous un prétexte fallacieux? En fait, "femmes et petits enfans » colportent par leur ingénuité la lettre de la violence ligueuse. C'est l'insertion dans le texte de d'Aubray qui permet de dénoncer cette propagande et impose une lecture ironique (une «plaisante rime », p. 96). Par conséquent, ni la harangue ni le libelle ne présentent la voix du peuple parisien comme la voix de l'intérêt général susceptible d'être à l'origine de l'autorité du pouvoir ${ }^{11}$. Le libelle ne participe donc pas du mouvement insurrectionnel. En revanche, par quelle autorité l'ironie de d'Aubray rétablit le sens des mots, c'est ce que nous allons essayer de comprendre.

Écrit de circonstance, le libelle attaque par le rire le principe même des états généraux et de l'élection d'un roi. En effet, le libelle Abbrege et l'Ame des Estatz convoquez à Paris première version de la Satyre Menippee - est diffusé en «copies à la main » pendant la réunion des états généraux qui s'ouvrent à Paris le 26 janvier 1593. Ces états généraux visent à contrer l'accession au trône du roi naturel, Henri de Navarre. Réunis par le «Lieutenant de l'État et de la couronne ${ }^{12}$ » Mayenne, ils ont pour propos l'élection d'un roi. Le libelle défend au contraire la souveraineté du roi, dans la lignée de la doctrine de la monarchie absolue fixée par Jean Bodin ${ }^{13}$ : tout discours œuvrant à promouvoir une candidature au trône est d'emblée illégitime en droit, donc ridicule avant même la caricature amplifiante. Ainsi, outre des pièces importantes en encadrement (dont deux discours prêtés à l'imprimeur), le recueil se compose de six parodies burlesques de l'éloquence des ligueurs aux états généraux et, prêtée à un délégué du tiers état, Claude d'Aubray, d'une harangue fleuve polémique mais grave qui s'achève sur un panégyrique d'Henri de Navarre. Les six premiers discours, à travers le soutien apporté à différentes candidatures à la royauté, tournent au dénigrement par antiphrase des orateurs et de l'élection. La dernière harangue, véritable catilinaire, défend la reconnaissance du roi légitime «pour representer l'interest que chacun a d'avoir la paix » (p. 110). Le rire politique de la Satyre Menippee fixe un ridicule inspirant crainte et pitié.

Dans les faits, l'assemblée devient sans objet puisque Henri de Navarre annonce le 17 mai 1593 qu'il abjure. Après le sacre du roi à Chartres le 27 février 1594, Paris capitule et accueille finalement le roi le 22 mars suivant ${ }^{14}$. La Satyre Menippee aurait donc dû être caduque après la capitulation de Paris en mars 1594. Or son succès perdure : la version que nous lisons date de 1595 et les réimpressions régulières parues du XVI siècle à nos jours l'attestent. Le libelle semble être devenu un modèle de parole politique sécularisée, opposant la résistance du rire et de la raison aux excès du discours théologico-politique.

\footnotetext{
${ }^{11}$ La notion de vox populi semble apparaître quelques années plus tard. M.-M. Fragonard (dans « Obscurs, sans grade, fous et diffamés : les voix du peuple des pamphlets », J. LECOINTE, C. MAGNIEN, I. PANTIN et M.Cl. Thomine (dir.), Devis d'amitié. Mélange en l'honneur de Nicole Cazauran, Paris, Champion, 2002, p. 867885) note deux occurrences en 1617 (p. 870).

12 « Tiltre inouy, et estrange, qui a trop longue queuë, comme une chimere contre nature qui fait peur aux petits enfans » (p. 102), «qualité [...] qui sent plus à la verité le stile d'un clerc de palais, ou d'un pedant, que la gravité de la charge » (éd. cit., p. 112), lit-on dans la harangue de d'Aubray, qui discute la conformité juridique de ce titre.

${ }^{13}$ MoRel H., L'Idée gallicane au temps des guerres de Religion (1944), Aix-en-Provence, Presses universitaires d'Aix-Marseille, 2003, p. 103-104, et l'ensemble du chapitre II, p. 99-118.

${ }^{14}$ Les Entrées royales et solennelles du règne d'Henri IV dans les villes de France. I. 1594-1596, éd. M.F. WAGNER, Paris, Classiques Garnier, 2010, p. 157-206.
} 
Délégué du tiers état, délégué d'une bonne ville

L'attribution de la Satyre Menippee fait l'objet d'une enquête dans les parodies de paratextes prêtés à l'imprimeur (p. 5 et 157). Ce dispositif d'anonymat, souligné sur le mode comique, dissimule l'origine du libelle parmi les positions en conflit. L'énonciation de fiction est ainsi déléguée pour un temps à une figure historique considérée comme « Politique ».

Claude d'Aubray parle en fait en délégué du tiers état, c'est-à-dire, avant tout, en délégué d'une bonne ville ${ }^{15}$. Il affirme une identité et une fierté de Parisien : «Je suis amy de ma patrie, comme bon bourgeois et citoyen de Paris », lit-on (p. 114). Rappelons que par étymologie le bourgeois « vit dans un bourg » tandis que le citoyen "participe aux institutions d'une cité ». En outre, tout comme l'appartenance à la partie inclut celle au tout, ce sentiment d'appartenance à une ville se combine au sentiment d'appartenance à une terre natale ou patrie, c'est-à-dire « le pays du père ». Le personnage précise qu'il a été " eschevin et prevost des marchands en ceste ville, du temps qu'on y procedoit par libre eslection, et qu'on ne forçoit ny violentoit personne pour les voix et suffrages » (p. 79), soit avant l'insurrection des Seize. Né à Paris et élu pour deux ans, le prévôt des marchands surveille l'exécution des ordres du roi et des arrêts du parlement ${ }^{16}$. La municipalité parisienne a de larges attributions : contrôle du commerce fluvial sur la Seine (navigation et circulation des marchandises), commande de la milice bourgeoise et contrôle des remparts et des portes, justice pour les causes qui relèvent de sa police, gestion des rentes sur l'hôtel de ville ${ }^{17}$. Par conséquent, le délégué du tiers état d'Aubray parle au nom de deux appartenances : patriote, il se prononce contre l'impérialisme des Espagnols et des Lorrains; Parisien, il plaide à charge et à défense pour et contre Paris.

Quel est le pouvoir de représentation d'un délégué d'une bonne ville ? "J'auroy honte de porter la parolle pour ce qui est icy du tiers estat, si je n'estoy bien advoüé d'autres gens de bien qui ne se veullent mesler avec ceste canaille », dit le personnage de d'Aubray (p. 111). Le pronom «nous » représente en fait une population parisienne aux limites variables. Si le «nous » liminaire déplore devant les états réunis la misère des Parisiens (p. 75), le «tu » accusateur qui lui succède condamne la ville pour sa sédition (p. 76). La harangue évoque à maintes reprises en termes péjoratifs la «simple populace» (p. 88), le «peuple » que l'on « persuade » (p. 101) et que l'on «trompe» (p. 94) par une éloquence duplice, capable de « fureurs populaires » (p. 109). L'intérêt parisien que représente d'Aubray prend ses distances avec la sédition du «menu peuple» (p. 85) : la harangue exclut tout un petit peuple urbain de la représentation par le pouvoir municipal, ce qui permet en retour de comprendre l'adhésion de cette même population urbaine à un discours concurrent, revendiquant l'autonomie municipale. En revanche, le délégué défend le «public » contre des intermédiaires spoliateurs et pillards ${ }^{18}$, ou même contre la «racaille » parlementaire conjurée (p. 78) et les «quoquins » que sont les Seize (p. 114). Le personnage de d'Aubray parle en ancien membre du Bureau de la ville, c'est-à-dire avec la préoccupation d'un bien commun. Ce bien commun suppose la

\footnotetext{
${ }^{15}$ HAMON P., « Bonnes villes », La France de la Renaissance. Histoire et dictionnaire, A. JOUANNA ET AL. (dir.), Paris, R. Laffont, 2001, col. 642b à 644b.

${ }^{16}$ Franklin A., Paris et les Parisiens au XVI siècle. Paris physique. Paris social. Paris intime, Tours, ÉmilePaul frères éditeurs, 1921, p. 77. Sur Paris au XVI ${ }^{\mathrm{e}}$ siècle, pendant les guerres de Religion ou sous la Ligue, voir aussi BABElon J.-P., Nouvelle Histoire de Paris. Paris au XVI siècle, Paris, Hachette, 1985 ; DIEFENDORF B. B., Paris City Councillors in the Sixteenth Century : The Politics of Patrimony, Princeton, Princeton University Press, 1983, première partie : «Portrait of a Municipal Elite », p. 3-80.

${ }^{17}$ DESCIMON R., «L'échevinage parisien sous Henri IV (1594-1609). Autonomie urbaine, conflits politiques et exclusives sociales », N. BULST et J.-Ph. GENET (dir.), La Ville, la bourgeoisie et la genèse de l'État moderne, Paris, Éditions du CNRS, 1988, p. 113-150, ici p. 119.

${ }^{18}$ Éd. cit., p. 118 : «Nous n'aurons plus ces sangsuës d'exacteurs, et maletostiers : on ostera ces lourds imposts qu'on a inventé à l'hostel de ville sur les meubles et marchandises libres, et sur les vivres qui entrent aux bonnes villes, où il se commet mille abuz et concussions, dont le proffit ne revient pas au public, mais à ceux qui manient les deniers. »
} 
participation aux charges de la ville (impôts, milice), mais aussi les droits de bourgeoisie que donne la résidence à Paris. Il défend les intérêts d'une bourgeoisie marchande, engagée dans une logique capitaliste. Il rappelle la légitimité d'une autre institution malmenée pendant l'insurrection, le parlement de Paris ${ }^{19}$. Il défend enfin la souveraineté du roi, comme dans cette invective à l'encontre de Mayenne, dans laquelle il condamne l' ' advancement de vostre pere et de vos oncles [...] qui avoyent envahy et usurpé toute l'authorité et puissance Royale du temps du petit Roy François leur neveu » (François II) (p. 79). Au plan éthique, l'ancien prévôt des marchands se présente comme un ligueur repenti (p. 79) : attaché à la coutume, au christianisme de ses pères, c'est à ce titre que d'Aubray démystifie la trahison du peuple parisien manœuvré par l'éloquence duplice de la Ligue. Le point de vue polémique prêté à d'Aubray se définit par l'adhésion aux institutions municipales antérieures à la crise. En revanche, il représente une oligarchie parisienne « ralliée » ou en voie de ralliement grâce au libelle lui-même.

Retrouvant la voix de l'hôtel de ville antérieure à l'insurrection, D'Aubray réactualise ainsi la voix d'un «pouvoir par essence médiateur », qui sert d'intermédiaire entre les Parisiens et le souverain $^{20}$ : le discours renoue avec la loyauté traditionnelle des bonnes villes envers la monarchie. Ce dispositif de fiction sert dans les faits l'expression monarchique d'un milieu de parlementaires parisiens gallicans ${ }^{21}$.

\section{En l'absence du roi, le conflit des représentations théologico-politiques}

À Paris, en mai 1588, la rébellion a chassé Henri III. S'ensuit de mai à août 1590 le très rude siège de la forteresse par les troupes d'Henri de Navarre : la capitulation de Paris soulève alors le problème du retour au loyalisme envers la monarchie des Valois-Bourbons. Le conflit se déplace à ce moment du rapport de force armé à la polémique verbale. Dans ce contexte, la harangue dénonce les représentations spirituelles diffusées par la Ligue et œuvre à en substituer de nouvelles, à travers une écriture séculière de l'histoire.

La relecture de l'histoire parisienne récente désavoue le Paris ligueur et les «coups du ciel » de l'éloquence ligueuse. Dans le texte de d'Aubray, l'interprétation de l'histoire se tient en effet dans les limites de la nature humaine et repose sur le jeu des passions. Le modèle semble être l'écriture de l'histoire selon Tacite :

J'ay bonne memoire du commencement de la querelle qui vint entre feu monsieur vostre pere, et feu monsieur le Connestable, laquelle ne proceda que de jalousie de l'un sur l'autre. [p. 79] Le bien publiq estoit le charme et ensorcelement qui bouchoit l'aureille à nos predecesseurs : mais l'ambition et la vengeance de ces deux grandes maisons en estoit la vraye, et primitive cause, comme la fin le descouvrit. [p. 109]

L'analyse ôte au conflit son caractère confessionnel : selon elle, le point de départ des guerres civiles, ce sont des appétits du corps, des rivalités personnelles et féodales. La menace du schisme dans l'Église d'Occident n'a été qu'un instrument au service d'intérêts de grands seigneurs. La sécularisation du récit historique s'étend à l'exposé. La composition du récit repose sur la recherche des causes et sur l'exposé de leur enchaînement. Le récit supprime donc toute rupture de cohérence qui pourrait laisser place à une lecture spirituelle supposant

\footnotetext{
${ }^{19}$ Éd. cit., p. 78 : «Où est la majesté et gravité du parlement et jadis tuteur des Roys, et mediateur entre le peuple et le prince ? vous l'avez mené en triomphe à la bastille, et trainé l'authorité, et la justice captive plus insolemment, et plus honteusement que n'eussent faict les Turcs. »

${ }^{20}$ DESCIMON R., art. cit., p. 117.

${ }^{21}$ L'on attribue la rédaction de la harangue de d'Aubray à Pierre Pithou (1539-1596) : MARTIN M., «La contribution de Pierre Pithou à la Satyre Ménippée. L'illustration paradoxale d'une conception exigeante du talent oratoire », M.-M. Fragonard et P.-E. LEROY (dir.), Les Pithou. Les lettres et la paix du royaume, Paris, Champion, 2003, p. 215-228.
} 
une intervention surnaturelle. L'éloquence du ligueur repenti conserve toutefois une compréhension chrétienne de l'action humaine. Comme l'indique la mention polémique suivante, les actes humains supposent le libre arbitre et la suffisance relative de l'homme, grâce à la faculté de la raison :

En fin puis que nous convenons, et nous raportons en tant de rencontres à la Cité de Hierusalem, que pouvons nous attendre autre chose, qu'une totale ruine, et desolation entiere comme la sienne, si Dieu par un miracle extraordinaire ne nous redonne nostre bon sens ? [p. 108]

Ce fragment exclut de lire l'accomplissement d'une providence dans les désordres qui forment l'histoire récente. Mais pour l'ancien prévôt des marchands, la grâce divine intervient en manière de synergie, pour restaurer la raison chez les hommes. En définitive, le récit de l'histoire de Paris, à travers laquelle la population de la ville scelle son identité collective, permet de préserver l'idéal d'unité entre les habitants.

Dans ce contexte, à l'encontre de l'éloquence ligueuse ${ }^{22}$, ce n'est pas la Jérusalem céleste des écrits prophétiques qui sert d'exemple (exemplum) pour l'éloquence politique. Soulignons avec William McCuaig et Pauline Smith $^{23}$ que c'est l'exemple profane de la Jérusalem décrite par l'historien Flavius Josèphe qui est mobilisé, avec ses aspects démographiques et matériels :

Vous sçavez tous en quel gouffre et abysme de desolation nous avons esté par ce long et miserable siege : et si ne le sçavez, lisez l'histoire de Josephe, de la guerre des Juifs, et du siege de Jerusalem par Titus, qui represente au naif celui de nostre ville. Il n'y a rien au monde qui se rapporte tant l'un à l'autre, comme Hierusalem, et Paris, excepté l'yssuë et la fin du siege : Jerusalem estoit la plus grande, et la plus riche, et peuplee vile du monde ? Aussi l'estoit Paris,

Qui eslevoit son chef sur toutes autres villes,

Autant que le sapin sur les bruyeres viles ${ }^{24}$.

Hierusalem fit mourir son Roy : et son oinct de la race de David, et le fit trahir par un de ses disciples, et de sa nation : Paris a chassé son Prince, son Roy, son oinct naturel, et apres l'a faict assaciner et trahir par un de ses moynes. [p. 106]

De manière polémique, les théologiens de la Sorbonne sont assimilés aux chefs des prêtres, puisqu'ils ont délié les sujets de leur fidélité au roi, et Henri III est assimilé au Christ. Le récit de d'Aubray décrit ensuite la division religieuse des Juifs dans Jérusalem, telle que la rapporte l'historien et témoin Flavius Josèphe. Enfin, l'empereur romain Titus est proposé en analogie à Henri de Navarre. Le récit de l'histoire prêté à d'Aubray se modèle sur celui de Flavius Josèphe : d'Aubray reprend à son compte le jugement très négatif porté sur le parti religieux des zélotes. Cet exemple rhétorique concourt donc à renforcer l'adhésion au pouvoir temporel. Il ne participe pas d'une représentation sublimée de Paris, mais contribue au tableau réaliste de la misère de Paris.

L'exposé historique prêté à d'Aubray prend pour modèle, semble-t-il, une lecture

\footnotetext{
${ }^{22}$ YARDENi M., «Le mythe de Paris comme élément de propagande à l'époque de la Ligue », Paris et Ile-deFrance. Mémoires publiés par la Fédération des sociétés historiques et archéologiques de Paris et de l'Ile-deFrance, Paris, t. XX, 1969, p. 49-63 ; CROUZET D., Les Guerriers de Dieu. La violence au temps des troubles de religion, vers 1525 - vers 1610, Seyssel, Champ Vallon, 2005, vol. II, notamment chap. 17 et 18.

${ }^{23}$ McCuaig W., "Paris / Jerusalem in Pierre de L'Estoile, the Satyre Ménippée and Louis Dorleans », Bibliothèque d'humanisme et Renaissance, t. LXIV, $\mathrm{n}^{\circ} 2$ 2, 2002, p. 295-315, en particulier p. 307-310; SMith P. M., «The Reception and Influence of Josephus's Jewish War in the Late French Renaissance with Special Reference to the Satyre Menippee », Renaissance Studies, 13, 1999, p. 173-191.

${ }^{24}$ Traduction des Églogues de Virgile (I, 24-25), laquelle permet d'introduire la référence intermédiaire à Rome.
} 
antityrannique de Tacite, au service d'une pensée du maintien de l'État ${ }^{25}$. L'écriture tacitéenne de l'histoire permet l'affirmation, dans la Satyre Menippee, d'une pensée sécularisée du pouvoir, c'est-à-dire d'une pensée du politique. Toutefois, elle ne résout pas le problème de la souveraineté du prince.

La dernière harangue du libelle offre ainsi un exemple fictif d'éloquence politique sécularisée, c'est-à-dire chrétienne mais civile, dégagée du discours dévot et spirituel. Les ligueurs ont utilisé la revendication d'autonomie municipale pour entrer en conflit avec Henri III : la harangue de d'Aubray défend une option de ligueur repenti. La palinodie repose sur un paradoxe : la défense de l'intérêt de Paris se dissout dans l'adhésion au pouvoir monarchique réinstauré et à la personne d'Henri de Navarre, c'est-à-dire à une monarchie forte.

\section{Doléances aux états généraux : «Paris n'est plus Paris »²6}

La rédaction du libelle se modèle sur la pensée de Jean Bodin : loin de pouvoir élire un roi, les états généraux peuvent seulement exposer des doléances auprès du roi. La dernière harangue recourt ainsi à une rhétorique pathétique, maniant le regret et la nostalgie. D'Aubray veut «deplorer ce pitoyable estat de ceste Royne des villes, de ce Microcosme, et abrégé du monde » (p. 106). Rappelons que les privilèges de Paris ont été abolis par lettres patentes du roi en mai 1589 : cette perte, que la harangue ne mentionne pas explicitement, régit les doléances prêtées à d'Aubray.

\section{Des barricades à l'imaginaire du siège intérieur}

La harangue s'emploie à défaire l'imaginaire eschatologique et millénariste des ligueurs grâce à un tableau réaliste de la misère de Paris. Faisant fond sur des valeurs économiques et marchandes, le discours du ligueur repenti affiche ainsi une meilleure adéquation au réel, celui de la prospérité dans l'histoire récente. Habilement, il détourne toutefois le motif du siège militaire : le motif n'est plus imputé à charge à l'encontre d'Henri de Navarre, il est retourné en un siège métaphorique et intestin, pour le blâme de la sédition ligueuse.

Le tableau rhétorique de la misère de Paris, lieu commun des mémoires du temps ${ }^{27}$, est particulièrement amplifié : deux descriptions (p. 76-78 et 104-105) rappellent les violences physiques, la ruine matérielle des bourgeois, la corruption de la pensée. Par la description de la ruine des institutions et de l'arrêt de l'activité marchande, l'orateur cherche le trait réaliste :

Nostre hostel de ville que j'ay veu estre l'asseuré refuge du secours des Roys, en leurs urgentes affaires, est à la boucherie : nostre court de parlement est nulle : nostre Sorbonne est au bourdel, et l'université devenue saulvage. [p. 75]

Le discours de d'Aubray discerne dans l'histoire récente une révolution qui a mis à bas les

\footnotetext{
${ }^{25}$ Il conviendrait d'analyser en détail la portée du modèle de Tacite dans l'écriture de l'histoire qu'offre la Satyre Menippee. En particulier, il faudrait situer la conception du pouvoir dans la Satyre Menippee par rapport aux différentes pensées politiques issues de Tacite au XVI siècle : une telle étude outrepasse les limites du travail présent. Pour une telle analyse, nous renvoyons à la bibliographie due à A. E. Baldini, « Ragion di stato, tacitismo, machiavellismo e antimachiavellismo tra Italia e Europa nell'età della Controriforma. Bibliografia (1860-1999) », La Ragion di Stato dopo Meinecke e Croce. Dibattito su recenti pubblicazioni, Gênes, Name, 1999, p. 223-265. Nous avons particulièrement utilisé deux études de Rosanna Gorris, « Sotto il segno di Tacito, tra Italia e Francia », Alla corte del principe, Ferrare, Università degli studi di Ferrara, 1996, p. 235-262, et «"La France estoit affamée de la lecture d'un tel historien" : lectures de Tacite entre France et Italie », D. BOHLER et C. MAGNIEN-SimONIN (dir.), Écritures de l'histoire (XIV ${ }^{e}$-XVI $I^{e}$ siècle), Genève, Droz, 2005, p. 113-141.

${ }^{26}$ Abbregé et l'Ame des Estatz de Paris, dans Satyre Menippee, éd. cit., p. 512.

${ }^{27}$ Satyre Menippee, éd. cit., note 606, p. 335. La harangue de Rose a déjà décrit les collèges désertés par les professeurs et les étudiants, transformés en étables et écuries (p. 57-60).
} 
valeurs de Paris ${ }^{28}$. Il permet aussi de rétablir l'usage des mots en leur sens propre, puisque les signes sont ainsi garantis, au sens monétaire et fiduciaire, par leur adéquation au réel physique $^{29}$. Les deux descriptions établissent une antithèse entre un âge d'or, la prospérité antérieure entretenue par l'activité économique et marchande de la ville, et la ruine présente. L'éloge paradoxal de la prise de la ville (p. 104-105) permet aussi d'évoquer la prospérité à l'irréel du présent, c'est-à-dire de fantasmer la richesse matérielle possible si le siège n'avait pas eu lieu et si Henri de Navarre avait été accueilli par la ville. Ainsi, paradoxalement, la harangue avance des arguments réalistes tout en les nimbant de l'irréalité du souvenir nostalgique ou du fantasme d'un avenir non advenu. Aux images spirituelles des ligueurs, la harangue de d'Aubray substitue des images certes produites par la sensibilité humaine mais guère moins irrationnelles. Toutefois, le soin mis à rétablir le sens propre des mots permet de restaurer une communauté de parole dans laquelle le sens des mots est partagé de tous les Parisiens souffrants, quels que soient leur parti ou leur confession.

Un trait de cette rhétorique du monde renversé concerne ce qui fait l'orgueil d'une ville franche et la définit : les murs d'enceinte ${ }^{30}$. Par le jeu des métaphores, le discours de d'Aubray souligne l'inversion de fonction des fortifications de Paris. En effet, les murailles (élevées sous Philippe Auguste et Charles V) délimitent la cité et lui donnent son identité de ville franche. Toutefois, selon l'imaginaire métaphorique, au lieu de faire de Paris une zone de sécurité, elles ont favorisé l'insécurité intérieure. Dès la captatio benevolentice, l'orateur laisse notamment entendre que la construction des barricades, nouveauté de cet épisode d'insurrection urbaine, s'apparente à un siège intérieur :

Ce n'est pas sans cause que les autres nations nous appellent Caillettes, puis que comme pauvres cailles coyffées, et trop crédules, les prédicateurs et Sorbonistes, par leurs caillets enchanteurs, nous ont faict donner dans les retz des tyrans, et nous ont par apres mis en cages, renfermez dedans noz murailles pour apprendre à chanter. [p. 75]

Les Parisiens, «renfermez dedans [leurs] murailles », sont assimilés par métaphore à des cailles, gibier recherché par les chasseurs pour la consommation. L'orateur développe un tableau allégorique :

Nous sommes serrez, pressez, envahiz, bouclez de toutes parts, et ne prenons l'ayr que l'ayr puant d'entre nos murailles, de nos bouës, et égouts : Car tout autre ayr de la liberté des champs nous est deffendu. [p. 108]

Les Parisiens, prisonniers d'eux-mêmes comme des oiseaux dans une cage, sont privés de la campagne environnante, de leur liberté de mouvement et même de liberté métaphorique. La métaphore des tyrans en chasseurs fait des Parisiens animalisés, assimilés à des bêtes acculées, des victimes. L'allégorie est subtile et satirique : les Parisiens ont aussi été victimes d'eux-mêmes, puisque ce sont "prédicateurs et Sorbonistes » qui ont joué de l'appeau («caillets »). L'autorité des religieux et des théologiens, catholiques donc, a servi des intérêts personnels tyranniques. Par métaphore, l'éloquence des prédicateurs et des théologiens devient un chant fallacieux, mime trompeur de la parole authentique, qui dépossède la ville de sa propre voix («pour apprendre à chanter»). La harangue de d'Aubray s'emploie à

\footnotetext{
${ }^{28}$ L'Image du monde renversé et ses représentations littéraires et paralittéraires de la fin du XVI siècle au milieu du XVII siècle, J. LAFOND et A. REDONDO (dir.), Paris, Vrin, 1979.

${ }^{29}$ DEMONET M.-L., «Un aspect du discours polémique avant Les Provinciales: du bon sens des mots dans la Satyre Ménippée », Courrier du centre international Blaise Pascal, nº 18, 1996, p. 43-50.

${ }^{30}$ Aux XVI ${ }^{\mathrm{e}}$ et $\mathrm{XVII}$ e siècles, les murs d'enceinte offrent le « critère discriminant qui permet d'identifier la ville en la distinguant du bourg », rappelle Roger Chartier (Histoire de la France urbaine, G. DUBY (dir.), t. III, La Ville classique, de la Renaissance aux révolutions, E. LE ROY LADURIE (dir.), Paris, Seuil, 1981, p. 121).
} 
désaffubler la voix de Paris de l'aliénation ligueuse en retrouvant le sens conventionnel des mots. L'enjeu est le rappel d'une civilité urbaine perdue : on note que la culture de la voix, associée à la propagande des prédicateurs et à la sottise du menu peuple, est dévaluée, au profit d'un rétablissement du sens des mots, dans la Satyre Menippee, par l'écrit et l'imprimé, caractéristiques de la culture urbaine.

Ainsi, la harangue repose implicitement sur le lien étymologique entre vie de la cité et vie civile, ou encore sur le lien étymologique entre le mot grec signifiant «ville » (polis) et « police », mot qui désigne aussi bien le « gouvernement public » que l' " ensemble des règles du comportement social ». Or la ville de Paris, ruinée après l'insurrection et le siège, est décrite sous le jeu des images de la campagne ou de la forêt, de la nature sauvage. La Satyre Menippee dénonce ainsi le fait qu'à Paris, à la culture, à la civilité ou à la police urbaines ait succédé un état de nature barbare.

\section{Le roi, bourgeois de Paris?}

Le discours de doléances oppose la représentation empreinte d'orgueil de la place forte, entretenue par l'hôtel de ville, et la réalité d'une ville désormais ruinée par le heurt d'intérêts partisans. Pour la défense d'un idéal urbain médiéval et renaissant et alors qu'une déclaration de 1528 «fixe à Paris la résidence ordinaire du roi » ${ }^{31}$, la harangue fait ainsi du roi un bourgeois de Paris.

La présence étrangère achève en effet de trahir l'identité de la forteresse. Paris est décrit comme une ville cosmopolite, mais cet argument est négatif dans ce tableau du monde renversé. Dans une exclamation pathétique, la ville est dépeinte comme une place forte occupée :

O Paris qui n'es plus Paris, mais une spelunque de bestes farouches, une citadelle d'Espagnols, Ouallons, et Napolitains [...] [p. 75]

L'attaque contre la présence des troupes étrangères peut être lue comme un sursaut national, mais aussi, dans le même mouvement, comme la réaction indignée d'un Parisien face à l'abolition d'un privilège. Paris était en effet naguère exempté de la charge d'hébergement des troupes. La harangue oppose l'emprise des seigneurs de la Ligue et l'exercice du pouvoir par le roi :

Et ce fut lors que les Parisiens commencerent à voir des hostes vivants à discretion en leurs maisons, contre tous les anciens privileges, à eux accordez par les deffuncts Roys. [p. 98]

Selon l'attaque, Mayenne dessert l'intérêt de la ville, tandis que le roi conforte l'identité de Paris en accordant un privilège, en l'occurrence une exemption.

Dans une apostrophe à Paris et aux «villes libres », la harangue rappelle le statut de ville franche :

Apprenez donc, villes libres, apprenez par nostre dommage, à vous gouverner d'oresenavant d'autre façon : et ne vous laissez plus enchevestrer, comme avons faict, par les charmes et enchantements des prescheurs, corrompuz de l'argent, et de l'esperance que donnent les Princes, qui n'aspirent qu'à vous engager, et rendre si foibles, et si souples, qu'ils puissent joüyr de vous, et de vos biens, et de vostre liberté à leur plaisir. [p. 108]

À l'enrichissement vertueux par le commerce, la description oppose l'enrichissement vicieux par la corruption : le premier garantit l'autonomie municipale, le second conduit à la tyrannie des intérêts particuliers. Par contraste, la harangue décrit le roi comme le protecteur de Paris ${ }^{32}$.

\footnotetext{
${ }^{31}$ CHARTIER R., op. cit., p. 177.

${ }^{32}$ Voir aussi la description des tapisseries, éd. cit., p. 20 : « Mais au fond et milieu de la piece estoient figurees
} 
Le roi manifeste en effet sa libéralité en participant à ce qui est pourtant une charge de la ville, une marque de son autonomie, à savoir l'entretien et la modernisation des fortifications ${ }^{33}$ :

Tu [Paris] n'as peu supporter ton Roy si debonnaire, si facile, si familier, qui s'estoit rendu comme concitoyen, et bourgeois de ta ville, qu'il a enrichie, qu'il a embellie de somptueux bastiments, accreuë de forts et superbes ramparts, ornee de privileges et exemptions honorables. [p. 76]

Sur le plan juridique, la bourgeoisie suppose que l'on ait résidé au moins un an à Paris, que l'on possède des biens, que l'on s'acquitte des taxes et que l'on serve dans la milice. En participant aux charges liées aux remparts, le roi contribue aux charges qu'impose la résidence ou bourgeoisie à Paris. La lecture de l'histoire récente minore ainsi le motif du conflit entre la ville et le roi Henri III, grâce à un euphémisme : "Tu n'as peu supporter une légère augmentation de taille, et d'offices : quelques nouveaux edicts qui ne t'importoient nullement » (p. 76). En fait, alors que Paris est normalement exempté d'impôts directs, l'euphémisme désigne les demandes de trésorerie effectuées au cours des années 1570-1580, pour combler le déficit du budget royal. Notons que les arguments prêtés à d'Aubray indiquent plutôt, contre l'interprétation et l'usage qu'en fait la harangue, la mainmise que le pouvoir royal a exercée sur Paris avant le conflit, la fragilité de privilèges urbains en partie vidés de leur contenu et, de fait, la transformation de la ville en capitale du royaume.

Finalement, la harangue avance un argument en faveur du ralliement loyaliste à Henri de Navarre : par sa résidence au Louvre, le roi était «comme concitoyen, et bourgeois de ta ville », bref, Parisien. La dernière harangue de la Satyre Menippee exprime en fait une loyauté à un état passé du gouvernement de Paris :

Rather than a powerful, independant force defending its integrity against the assaults of the monarchy, the city government was in fact an agency of limited mandate, dependant for its very existence on the toleration that the kings accorded it for the sole reason that it served their own purposes well ${ }^{34}$.

L'hôtel de ville parisien protège certes les intérêts marchands et urbains, mais il n'agit en ce sens que dans la dépendance du pouvoir du roi.

\section{Misère et messianisme}

Ces doléances relèvent d'un discours globalement sécularisé, grâce au rappel de deux conventions sociales : le sens des mots et les privilèges municipaux. La description de la ville ruinée convoque néanmoins les références surnaturelles du salut : la royauté est sacrée, et le roi, un messie.

Le roi est en effet qualifié d' « oinct naturel »(p. 106, extrait cité supra). Comprenons, suivant Daniel Ménager, que «le caractère du roi préexiste à l'onction»lors du sacre ${ }^{35}:$ la personne du roi est sacrée avant l'institution religieuse du roi :

les barricades de Paris, où l'on voyoit un Roy simple et bon Catholique, et qui avoit tant faict de biens et donné tant de privileges aux Parisiens, estre chassé de sa maison, et assiegé de toutes parts, avec tonneaux et barriques pour le prendre. »

${ }^{33}$ CHEVALIER B., «L'État et les bonnes villes en France au temps de leur accord parfait (1450-1550)», La Ville, la bourgeoisie et la genèse de l'État moderne..., op. cit., p. 77.

${ }^{34}$ DIEFENDORF B. B., op. cit., p. 14.

${ }^{35}$ MENAGER D., «Dieu et le roi », art. cit., p. 216 ; ID., « L'image du prince dans la Satyre Menippee », L'Image du souverain dans les lettres françaises : des guerres de Religion à la révocation de l'édit de Nantes, N. HEPP et M. BeRTAUd (dir.), Paris, Klincksieck, 1985, p. 201-210, ici p. 205-206. 
Je dy vostre Roy : car je trouve emphase en ce mot, qui emporte une personne sacree, oincte, et cherie de Dieu, comme mytoyenne entre les Anges et les hommes : Car comment seroit-il possible qu'un homme seul, foible, nud, desarmé, peust commander à tant de milliers d'hommes, se faire craindre, suyvre, et obeyr en toutes ses voluptez, s'il n'y avoit quelque divinité, et quelque parcelle de la puissance de Dieu meslee ? [p. 99]

L'autorité du roi héréditaire est donc fondée à la fois en nature et en surnature. La doctrine théologico-politique du pouvoir royal légitime fonde la condamnation du régicide puis l'obéissance à Henri de Navarre, quelle que soit la confession de ce dernier. De la sorte, l'autorité du roi doit donc être reconnue avant l'éventuel sacre prévu par la religion :

Il n'y a plus lieu d'election, et faut accepter avec joye et alegresse ce grand Roy que Dieu nous envoye, qui n'a que faire de nostre ayde pour l'estre et qui l'est desjà sur nous, et le sera encor malgré nous, si nous l'en voulons empescher. [p. 123]

La harangue de d'Aubray prend soin de distinguer l'élection du roi et la reconnaissance du roi, ce qu'elle accomplit.

Rappelons que l'« oint », c'est en hébreu le «messie ». Or, dans l'ensemble de la harangue, par contraste avec la rhétorique zélée, l'emploi de références aux Écritures apparaît délibérément limité ${ }^{36}$. Cependant, une citation du Livre d'Isaïe introduit un discours messianique, l'annonce qu'arrive le libérateur de Jérusalem :

O quam speciosi pedes nuntiantium pacem : nuntiantium bona et salutem, dit Isaye. O que ceux ont les pieds beaux, qui portent la paix, et annoncent le salut et la sauveté des peuples. [p. 126]

Même si la référence est allusive, le contexte ne laisse aucun doute sur le fait que, pour Paris, l'homme providentiel est Henri de Navarre. Ce recours à la référence scripturaire renoue-t-il avec le discours chrétien spirituel des prédicateurs ligueurs ? Cette référence s'apparente à l'usage que l'on fait au XVI ${ }^{\mathrm{e}}$ siècle du prophétisme d'Isaïe dans les entrées de ville offertes au roi $^{37}$. D'Aubray utilise ce qui est devenu un lieu commun de la publicité faite au roi dans l'entrée solennelle à Paris, au cours du siècle : la harangue figure par anticipation ce rite d'accueil.

Après le désaveu de la journée des barricades qui a chassé le roi hors de Paris, puis celui du régicide, la harangue restaure symboliquement le pouvoir royal : elle figure l'entrée en ville d'Henri de Navarre, telle qu'elle conclue à nouveau l'alliance entre le roi et Paris et permette le retour du roi dans sa résidence du Louvre.

À travers la description de Paris comme place forte, c'est le motif de Paris «ville franche » qui s'affirme. L'adhésion à la monarchie se comprend à travers l'attachement aux privilèges urbains perdus. La harangue de d'Aubray, voix de l'hôtel de ville, invite ainsi à considérer le roi comme un bourgeois de Paris, participant aux charges de la bourgeoisie, donc pourvu de ses droits : l'argument prépare la transformation de la ville en capitale du royaume.

\section{«Un roi qui donnera ordre à tout ${ }^{38}$ » : cuisine ménippéenne et raison d'État}

Que le discours ligueur apparaisse comme déraisonnable par la mise en abyme et l'ironie n'implique pas que la dénonciation de la Satyre Menippee repose entièrement sur une

\footnotetext{
${ }^{36}$ VIGNES J., «Culture et histoire dans la Satyre Menippee », art. cit., p. 171-173.

${ }^{37}$ CHONE P., «Entrées, feux d'artifice et fêtes à programme en France », Spectaculum europaum. Theatre and spectacle in Europe, Histoire du spectacle en Europe (1580-1750), Wiesbaden, Harrassowitz Verlag, 1999, p. 663-679.

${ }^{38}$ Éd. cit., p. 119.
} 
rhétorique de la raison. En particulier, le discours de d'Aubray comporte des fragments de raisonnement juridique, mais il ne verse pas dans le traité théorisant l'État: l'éloquence municipale prêtée à d'Aubray est tout aussi poétique que l'éloquence spirituelle des ligueurs. La harangue utilise l'ancienne métaphore monarchique des deux corps du roi, selon laquelle le royaume forme un corps mystique analogique du corps propre du roi ${ }^{39}$. Toutefois, à la différence du discours ligueur, ce discours poétique est fondé en nature, et non en surnature. Ainsi, la métaphore physique permet de susciter une communauté de parole et d'intérêts, en somme une police et une politique. La réécriture de la métaphore du corps du roi impose en fait une représentation métaphorique de l'État : le texte suggère une doctrine de l'État fondée en droit naturel, donc différente de la raison d'État machiavélienne, et positive ${ }^{40}$. Finalement, la poétique du corpus ménippéen s'éclaire à la lumière des développements métaphoriques de la loi de nature : la hantise de dévoration des Parisiens entre eux suscite par analogie et déplacement un texte dérivatif, une fricassée comique, rassasiant et apaisant les appétits.

\section{Du corps des Parisiens à l'abstraction du corps politique}

La présence obsédante du corps souffrant dans les récits du siège de Paris, note Antoinette Gimaret, entre dans «des schémas explicatifs servant à rendre lisibles les événements historiques $^{41}{ }^{\prime}$. Dès le récit prêté à d'Aubray, quasi d'actualité, l'écriture de l'histoire avance en effet que le corps des habitants de la ville attend la protection providentielle par le corps du roi. Ainsi, Henri de Navarre est attendu non pas en roi qui fasse le salut des âmes mais en roi pour la santé des corps. À partir d'un substrat sans doute tacitéen, l'écriture de la harangue déplace la réflexion politique : cette dernière concerne moins l'exercice du pouvoir que la formation du corps politique et social qui s'ébauche par les institutions urbaines et l'accord entre le prince et ses sujets.

Les habitants de Paris ne sont plus protégés par les franchises urbaines. Ils ne sont donc en droit plus même maîtres de leur corps :

Il faut confesser que nous sommes pris à ce coup, plus serfz, et plus esclaves, que les Chrestiens en Turquie, et les Juifz en Avignon. Nous n'avons plus de volonté, ny de voix au chapitre. Nous n'avons plus rien de propre que nous puissions dire cela est mien. [p. 75]

La harangue déroule la liste des violences faites au corps, l'assassinat du roi par un Parisien, celui de parlementaires de Paris, l'emprisonnement des notables, le viol des femmes, la famine. Aux crimes commis contre les vivants, il faut ajouter les atteintes contre les morts, d'abord contre les corps des saints, par la profanation des reliques, puis par ce que JeanClaude Mühlethaler nomme le «banquet d'horreur ${ }^{42}$ » à partir des cadavres. Signe de barbarie, pendant le siège, on signale en effet des pratiques anthropophages :

\footnotetext{
${ }^{39}$ Kantorowicz E., Les Deux Corps du roi. Essai sur la théologie politique au Moyen Âge (1957), Paris, Gallimard, 1989 ; WEBER H., «L'analogie corps humain - corps social dans la pensée politique du XVI siècle », Analogie et connaissance. I. Aspects historiques, A. LICHNEROWICZ, F. PERROUX et G. GADOFFrE (dir.), Paris, Maloine, 1980, p. 139-145.

${ }^{40}$ Pour l'approche de l'État à travers la raison d'État, sans réduction de cette dernière à son sens machiavélien, voir GAUCHET M., «L'État au miroir de la raison d'État: la France et la chrétienté », Y.-Ch. ZARKA (dir.), Raison et déraison d'État. Théoriciens et théories de la raison d'État aux XVI et XVII siècles, Paris, PUF, 1994, p. 193-244 ; voir la discussion de cet article dans la livraison des Cahiers du Centre de recherches historiques,

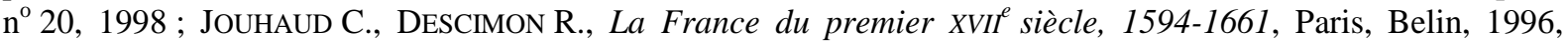
chap. 1.

${ }^{41}$ GIMARET A., Ordinaire et extraordinaire des Croix. Les représentations du corps souffrant, 1580-1650, Paris, Champion, 2011, p. 100-118, ici p. 102 ; ID., « La mémoire du corps souffrant ou la question de l'horreur. Les récits du siège de Paris de Goulart à Maimbourg », La Mémoire des guerres de Religion, op. cit., p. 193-209.

42 MüHLEthaler J.-C., «Des mets et des maux. Aspects et enjeux de la dévaluation de la table à la Renaissance », Romanische Forschungen, vol. 108, n 3/4, 1996, p. 396-424, particulièrement p. 415-419.
} 
Et n'a pas tenu à monsieur le Legat, et l'Ambassadeur Mandosse, que n'ayons mangé les oz de nos peres, comme font les saulvages de la nouvelle Espagne. [p. 77]

Pierre de L'Estoile mentionne aussi la fabrication de cette farine d'os ${ }^{43}$. La litote de la Satyre Menippee («Et n'a pas tenu à monsieur le Legat») est accusatrice : les intérêts étrangers achèvent de diviser le corps social et font tomber dans la barbarie de l'état de nature. Le motif de ce repas polémique, destiné à susciter crainte et pitié, peut être lu, suivant Mühlethaler, par différence avec la tradition du banquet philosophique. En effet, dans l'harmonie du banquet socratique, les propos de table signifient l'alliance du corps et de l'esprit. La prédication ligueuse apparaît au contraire comme une falsification spirituelle, puisque corps et esprit ne sont plus harmonieusement unis. Cette relation au corps, barbare, dénonce la «Sainte Union » que prétend être la Ligue : cette «Union» n'a pas d'existence spirituelle, selon la harangue prêtée à d'Aubray. La Ligue, prétexte d'intérêts particuliers, place le peuple parisien à la lisière de la culture et de la barbarie.

Contre le déploiement par la rhétorique ligueuse de tableaux allégoriques, figurant des réalités surnaturelles, la harangue substitue la description de réalités naturelles ramenant à la vie du corps. Le récit historique abonde en comparaisons triviales : empruntées à un fond moral populaire, les comparaisons introduisent des proverbes, des amorces de fables et rappellent l'obsession de la faim. Elles figurent la menace de la dévoration par un prédateur naturel ; par exemple :

Et le sçeutes si dextrement tourner et manier, que luy meistes une folle et indiscrette ambition dedans la teste, pour faire de luy comme le chat de la souriz, c'est à dire apres vous en estre joüé, de le manger. [p. 87]

Car ce qu'ils vous font entendre de la religion, n'est qu'un masque, dont ils amusent les simples, comme les renards amusent les pies de leurs longues queuës, pour les attraper et manger à leur ayse. [p. 108]

Il [le roi d'Espagne] ne nous engraisse pas pour nous vendre, comme les bouchers font leurs pourceaux [...]. [p. 116]

Nous ne voulons pas faire comme les grenouilles, qui s'ennuyans de leur Roy paisible, esleurent la Cigoygne qui les devora toutes. [p. 119, nous soulignons]

La loi de nature fait entrer le corps dans la chaîne alimentaire. Ces comparaisons dégagent la souffrance physique et les atteintes contre le corps de toute lecture sublimée ou spirituelle : les Parisiens ne sont pas des martyrs. Le propos politique mobilise des images du corps qui dessinent à la fois le corps de la population mourante et un corps métaphorique :

La religion Catholique et Romaine est le breuvage qui nous infatue, et endort : comme une opiate bien sucree, et qui sert de medicament narcotique, pour stupeffier nos membres, lesquels pendant que nous dormons, nous ne sentons pas qu'on nous coupe piece à piece, l'un apres l'autre, et ne restera que le tronq qui bientost perdra tout le sang et la chaleur, et l'ame, par trop grande evacuation. [p. 110]

La défense du corps spirituel qu'est l'Église, menée par la Ligue, apparaît par le jeu des images comme une menace pour le corps politique qu'est Paris. Les images du corps de la population permettent de délimiter le domaine du politique en un sens réaliste : les désordres touchant la monarchie, notamment l'éviction du roi naturel, atteignent les corps, donc corrompent les mœurs et par suite la religion.

Le domaine du politique apparaît nettement séparé des préoccupations spirituelles du salut des âmes : le souci du corps de la population urbaine. Les représentations, exploitant l'expérience

\footnotetext{
${ }^{43}$ PierRe De L'Estolle, Journaux-Mémoires, éd. G. Brunet ET AL., Paris, Librairie des bibliophiles, 1878, p. 26 (15 juin 1590).
} 
récente de la famine, fixent au politique la charge d'établir une frontière entre la vie barbare et la vie civile, afin que les habitants et sujets ne s'entredévorent, au sens littéral comme au sens métaphorique et moral. S'il serait excessif de dire que le roi doit faire le bonheur matériel de ses sujets, le pouvoir souverain du roi apparaît dans la Satyre Menippee comme la garantie de la prospérité urbaine.

\section{Le roi privé de son corps}

Le motif paulinien du corps mystique, désignant l'Église, circule du discours zélé où il désigne la «Sainte Union » au discours séculier de l'État prêté à un représentant de l'hôtel de ville parisien : la santé et la prospérité des corps sont fantasmées à travers la restauration du corps du roi. L'analogie ainsi établie entre corps du roi et corps des Parisiens explique les troubles civils, rend désirable la paix et fonde selon la loi de nature une doctrine de l'État.

L'analyse de l'éviction d'Henri de Navarre de la succession à la couronne établit une analogie entre la spoliation dont les Parisiens sont victimes - de leur corps, de leurs biens - et la spoliation du roi - de son bien, donc de son corps. Le passage suivant s'ouvre sur l'hypothèse où Henri de Navarre «persisteroit » dans la Réforme :

Mais quand ainsi seroit qu'il persisteroit en son opinion, pour cela le faudroit-il priver de son droict legitime de succession à la couronne ? Quelles loix, quels chapitres, quelle evangile nous enseigne de deposseder les hommes de leurs biens, et les Roys de leurs Royaumes pour la diversité de religion? l'excommunication ne s'estend que sur les ames, et non sur les corps, et les fortunes: Innocent troisiesme exaltant le plus superbement qu'il peut sa puissance Papale, dit que comme Dieu a fait deux grands luminaires au ciel, sçavoir est le Soleil pour le jour, et la Lune pour la nuict : ainsi en a-il fait deux en l'Église : l'un pour les ames, qui est le Pape, qu'il accompare au soleil, et l'autre pour les corps, qui est le Roy : ce sont les corps qui joüissent des biens, et non pas les ames : l'excommunication ne les peut oster : car elle n'est qu'un medicament pour l'ame, pour la guerir, et ramener à santé, et non pour la tüer : elle n'est pas pour damner, mais pour faire peur de damnation. [p. 121-122, les italiques sont de notre fait]

Le passage est complexe car le mot «corps », répété trois fois, change de sens : en l'absence de lien logique et parce qu'il interrompt un syllogisme, le passage que nous avons mis en italique apparaît comme une interpolation indépendante du tissu dans lequel elle est insérée. Le fragment interpolé énonce une stricte séparation des pouvoirs et des domaines d'autorité entre le spirituel et le temporel, d'où le royaume sort sécularisé. Précisons quelques enjeux juridiques. Le pape, d'une part, dont l'autorité s'exerce sur les âmes en vue de leur salut, contrôle l'Église : le pape n'est donc pas une médiation pour la souveraineté du roi de droit divin. Le royaume, d'autre part, est avant tout une réalité matérielle, le corps du sujet : l'État sécularisé protège les corps, c'est-à-dire autant les sujets que les objets. En revanche, le roi de France n'a pas d'autorité sur l'Église et peut donc être de confession différente. En outre, cette autorité sur les corps paraît compatible avec la division confessionnelle des sujets, puisque l'autorité perdurerait même avec un roi hérétique relaps. L'ancienne métaphore du corps mystique du roi - le royaume - est sécularisée : seul le roi demeure sacré, comme nous l'avons déjà noté. Par-delà la division confessionnelle, le motif du roi « pour les corps » a une puissance réconciliatrice et unifiante.

Ce fragment est intercalé dans un raisonnement juridique délimitant la portée de l'excommunication. Cette dernière ne peut pas empêcher la transmission du patrimoine par héritage : en effet, selon le syllogisme, l'excommunication ne concerne pas les corps, or les corps jouissent des biens, donc l'excommunication ne concerne pas les biens du roi, c'est-àdire les corps sur lesquels il doit régner. Selon la métaphore, le roi serait possesseur d'un bien, sur lequel l'Église ne saurait exercer son autorité : il s'agirait donc de remettre au roi le corps ou bien dont il est spolié, c'est-à-dire le royaume. Apparaît la notion d'État inaliénable. 
Conséquence capitale, en écartant l'intervention du pouvoir spirituel dans le domaine politique, la théorie de la monarchie qui sous-tend le texte dessine un pouvoir absolu, c'est-àdire sans partage : «Nous aurons un Roy qui donnera ordre à tout» (p. 119). Enfin, l'image du corps permet d'assimiler la relation que le roi entretient avec son propre corps physique à celle qu'il entretient avec le corps du royaume : la relation d'autorité du roi sur le royaume est comprise comme une relation de propriété, de possession. Le roi n'est donc pas au-dessus du royaume et de la loi. Le royaume sécularisé est en fait l'État, propriété d'une lignée de rois héréditaires, de droit divin.

Dans cette fondation de l'État par le droit naturel, on relève une contradiction : la harangue insiste sur le motif de l'hérédité du roi, à travers le rappel de la notion de "prince de sang », mais une loi positive vient cependant restreindre ce modèle biologique, la loi salique :

Vrayement, si nous n'avions plus de sang de ceste noble famille Royale, ou que nous fussions en un Royaume d'election, comme en Pologne, ou en Hongrie, je ne dy pas qu'il n'y fallust entendre : mais ayans de temps immemorial ceste loüable loy, qui est la premiere et la plus ancienne loy de nature, que le fils succede au pere, et les plus proches parens en degré de consanguinité à leurs plus proches de la mesme ligne et famille : et ayant un si brave et genereux Prince en ce degré, sans controverse ny dispute, qu'il ne soit le vray naturel et legitime heritier, et plus habile à succeder à la couronne : Il n’y a plus lieu d'election [...] [p. 123]

La loi salique est dite paradoxalement la «premiere et la plus ancienne loy de nature »: le raisonnement naturalise une convention. Le pouvoir absolu du roi n'exclut donc pas l'existence de lois fondamentales.

À ce moment du travail symbolique de l'écriture enrôlée, l'alliance entre la ville et le roi n'est donc pas figurable comme l'union de deux corps, celui du roi et celui des sujets. Le droit naturel est transgressé par la sédition parisienne et l'imposture ligueuse. La pensée politique est ainsi exposée au moyen d'une métaphore biologique, le « roi naturel », par opposition avec un « roi artificiel »:

Nous demandons un Roy et chef naturel, non artificiel : un Roy desja faict, et non à faire [...] le Roy que nous demandons est desjà fait par la nature, nay au vray parterre des fleurs de lyz de France : jetton droit et verdoyant du tige de S. Loys. [...] on peut faire une maison, mais non pas un arbre, ou un rameau verd : il faut que nature le produise par espace de temps du suc, et de la mouëlle de la terre, qui entretient le tige en sa seve et vigueur. On peut faire une jambe de bois, un bras de fer, et un nez d'argent : mais non pas une tête. [...] Aussi un Roy electif et artificiel ne nous sçauroit jamais voir, et seroit non seulement aveugle en nos affaires : mais sourd, insensible et immobile en nos plainctes. [p. 119]

La métaphore du roi artificiel désigne le burlesque tragicomique d'un roi élu par l'assemblée des états généraux : son éventuel pouvoir est assimilé à celui d'un estropié, voire d'une marionnette désarticulée. Le corps métaphorique du roi élu serait aussi mutilé que le corps réel des Parisiens ${ }^{44}$. De la sorte, la rhétorique des doléances aux états généraux perdrait toute efficacité sur un roi au corps insensible, incapable de perception, de passion et de compassion. L'analogie entre corps des habitants de Paris et corps du roi, confondus dans le corps de l'État, établit des correspondances horizontales : sans échappée surnaturelle, l'imaginaire cosmologique se réduit alors à l'inscription du corps du sujet dans le corps de l'État. L'imaginaire biologique qui affleure dans l'écriture rejoint la tradition néostoïcienne et

\footnotetext{
${ }^{44}$ Voir aussi la gravure dans l'édition de 1600 commentée par Anne-Marie Brenot, « Le corps pour royaume. Un langage politique de la fin du XVI $I^{\mathrm{e}}$ siècle et début du XVII ${ }^{\mathrm{e}} »$, Histoire, économie et société, $10^{\mathrm{e}}$ année, $\mathrm{n}^{\mathrm{o}} 4,1991$, p. 441-466.
} 
renvoie au motif de la loi de nature. La notion de loi de nature permet ainsi de fondre la tyrannie des intérêts particuliers en une loi de l'intérêt général : c'est cet ordre juridique que la harangue présente comme proprement biologique, par la fusion du corps des sujets et du corps du roi dans l'État. C'est en ce sens, à travers la postulation d'une loi de nature commune à tous, que la harangue suppose l'idée d'une raison d'État positive : le royaume est un État fondé en droit naturel, tandis que le prince tire sa souveraineté du droit divin. L'exemple des habitants de Paris permet de donner une forme rhétorique et poétique au corps politique.

La poétique de la Satyre Menippee est adéquate à la situation sociale et politique. La fricassée des formes, genres, tons, dont le lecteur se délecte, correspond à la division du corps politique et aux mutilations du corps des sujets dans la guerre civile. La métaphore alimentaire de ce corpus polémique fait écho à la violence des rapports de prédation entre les sujets du roi. À l'union dans l'eucharistie, autour du corps du Christ, suivant les ligueurs, la Satyre Menippee substitue par analogie un banquet comique, un repas fédérant selon les passions et appétits du corps et purgeant en vue de l'apaisement et de la maîtrise des mêmes passions. Appelant à la paix par la restitution du corps du royaume au roi légitime, la satyre ménippée - comprise comme genre - contient sa propre abolition : avec l'unité de l'État doivent prendre fin et le ridicule des prétentions partisanes et la parole satirique. Le genre du libelle s'abolit d'autant plus avec la Satyre Menippee que, d'après elle, le bien public ne saurait être défini par l'opinion publique.

\section{Conclusion}

Morceau dans la fricassée ménippéenne, la description de la forme de Paris participe d'un travail de distinction du spirituel et du temporel, de l'Église et de l'État, de la cité des hommes et de la cité de Dieu. La description métaphorique de Paris, proprement poétique, défait l'imaginaire ligueur, qui affublait la ville d'une métaphore désormais réservée à l'Église, la Jérusalem céleste. Elle vise à faire de la place forte et ville franche un membre du corps de l'État, au service du Conseil du roi. La topographie politique qui apparaît dans la harangue de d'Aubray, en particulier dans les deux déplorations de la misère de Paris, mais aussi dans le « Recueil poétique » qui suit, fait de Paris le «microcosme et abrégé » de l'État en formation.

L'idée nouvelle d'État est nettement figurée sur le modèle, sécularisé, de l'Église : la seconde a la charge des âmes, en vue de leur salut spirituel, le premier celle des corps des sujets et de leurs biens, en vue de leur protection et de la prospérité. Compte tenu de cette distinction des pouvoirs, qu'est-ce qui définit dès lors l'appartenance d'un corps à l'État ? Le discours de d'Aubray attire l'attention sur l'alliance de la ville de Paris avec le roi. La ville donne l'exemple de la reconnaissance par la municipalité et la population urbaine de la souveraineté du roi et de l'État. L'expérience du bien public ou de l'intérêt commun à l'intérieur de la ville franche semble transposée au plan de l'État : ce dernier devient lui-même associé à un public et un sens du bien commun. La harangue prêtée à un Parisien insiste en particulier sur les valeurs économiques et marchandes, mais aussi sur les valeurs d'une vie civile, contenant la violence interpersonnelle qui découle du conflit des intérêts particuliers. Dans l'idée d'État, ce sens du bien commun, qui retrouve l'idée antique de chose publique (res publica), réinstauré à travers la cité, s'associe donc à un sentiment d'appartenance à une nation et une patrie.

Le soutien de Paris au roi de France, figuré dans la Satyre Menippee et effectif par la suite dans l'histoire, prépare et autorise le centralisme de la monarchie absolue. Est-ce à partir de la transformation parisienne que les mots de «bourgeoisie » et de «citoyenneté » ont cessé de désigner un fait urbain pour nommer un fait national ? L'exemple de la Satyre Menippee permet du moins de comprendre comment a pu s'accomplir le glissement de ces valeurs de la 
ville franche dans le registre national ${ }^{45}$.

${ }^{45} \mathrm{Au}$ terme de ce travail, je remercie vivement Michèle Clément pour sa relecture et ses suggestions avisées. Ce qui est imparfait ou contestable demeure bien entendu sous ma seule responsabilité. 